\title{
Co-expression network analysis identified specific miRNAs and genes in association with slow-transit constipation
}

\author{
CHAORAN YU*, LU ZANG*, BO FENG, LUYANG ZHANG, PEI XUE, \\ JING SUN, FENG DONG, JUNJUN MA and MINHUA ZHENG \\ Department of Gastrointestinal Surgery, Shanghai Minimally Invasive Surgery Center, Ruijin Hospital, \\ Shanghai Jiao Tong University, School of Medicine, Shanghai 200025, P.R. China
}

Received February 22, 2020; Accepted May 29, 2020

DOI: $10.3892 / \mathrm{mmr} .2020 .11568$

\begin{abstract}
The pathogenesis of slow-transit constipation (STC) remains largely unclear, with the roles of microRNAs (miRs/miRNAs) yet to be determined. Co-expression network analysis of miRNAs in STC is crucial to elucidating potential underlying mechanisms. Weighted gene correlation network analysis was performed in the miRNA expression profile of STC (GSE57969). The key miRNA target genes were further functionally enriched by Kyoto Encyclopedia of Genes and Genomes (KEGG) and Gene Ontology (GO). A Protein-Protein Interaction (PPI) network was constructed, with a total of 12 color-clustered modules determined. Seven key miRNAs were established, including five miRNAs from the turquoise module (hsa-miR-20b, hsa-miR-128, hsa-miR-129-3p, hsa-miR-30b and hsa-miR-340), one miRNA from the blue module (hsa-miR-619) and one from the black module (hsa-miR-486-3p). A total of 2,077 key miRNA target genes were predicted. GO analysis revealed that the 'protein modification process' and 'cellular protein modification
\end{abstract}

Correspondence to: Professor Junjun Ma or Professor Minhua Zheng, Department of Gastrointestinal Surgery, Shanghai Minimally Invasive Surgery Center, Ruijin Hospital, Shanghai Jiao Tong University, School of Medicine, 197 Ruijin Er Road, Shanghai 200025, P.R. China

E-mail: zmhtiger@yeah.net

E-mail: marsnew1997@163.com

${ }^{*}$ Contributed equally

Abbreviations: STC, slow-transit constipation; WGCNA, weighted gene correlation network analysis; GO, Gene Ontology; KEGG, Kyoto Encyclopedia of Genes and Genomes; PPI, Protein-Protein Interaction; ICC, interstitial cells of Cajal; GEO, Gene Expression Omnibus; TOM, Topological overlap measure; GS, gene significance; BP, Biological Process; CC, Cellular Components; MF, Molecular Function; CALM2, calmodulin 2; CALM1, calmodulin 1

Key words: slow-transit constipation, weighted gene correlation network analysis, Gene Ontology, Kyoto Encyclopedia of Genes and Genomes, microRNA process' were the most significantly enriched processes in the 'Biological Processes' category, whereas the 'nucleoplasm' in 'Cellular Components' and 'enzyme binding' in 'Molecular Functions' were the most significantly enriched processes. The 'cAMP signalling pathway' was the top KEGG pathway. The hub genes identified from the PPI network included calmodulin (CALM)2, CALM1, histone deacetylase (HDAC)3, glycogen synthase kinase $3 \beta$, HDAC9, heat-shock protein family A member 8, G-protein subunit $\gamma$ (GNG)13, HECT domain and ankyrin repeat containing E3 ubiquitin protein ligase 1, GNG10 and GNG7. This bioinformatics analysis demonstrated co-expressed miRNA networks with insightful genes and pathways associated with STC.

\section{Introduction}

Constipation commonly affects $\sim 27 \%$ of the population in industrialized nations (1). It results in high frequencies of hospitalization, long-term overexposure to laxative prescriptions and a general healthcare burden worldwide $(2,3)$. Although constipation is not strictly gender-specific, a severe type is exclusively found in women (4). Slow-transit constipation (STC), affecting 13-37\% of patients with chronic constipation in both Western nations and Asia, commonly occurs in young women with a comparably long history of abnormal bowel movements since adolescence (2). For a number of patients with mild STC, a high-fibre diet alone can relieve the symptoms of constipation. Patients with severe STC, however, fail to respond to both dietary fibre and laxatives $(2,5)$. In China, the significance of STC has not been fully recognized. Limited therapeutic interventions result in increased excess laxative treatment, long-term discomfort and difficulty in obtaining samples $(6,7)$. In fact, the genomic investigation of STC remains sparse.

Notably, although multiple therapeutic management strategies, including fibre supplements, prokinetic drugs and biofeedback therapy, have been instituted, total colectomy and ileorectostomy remain the final and most effective management for patients with STC (2). In addition, there is an overlap between STC and other subtypes of constipation, including irritable bowel syndrome and pelvic floor dyssynergia, highlighting the sophisticated mechanisms involved in STC (8-10).

Previous histopathological investigations have revealed that a reduced number of interstitial cells of Cajal (ICC) and 
abnormalities in the myenteric plexus neurons, neurotransmitter $\mathrm{P}$ substance, intestinal peptide and nitric oxide are associated with STC (11-13). Interestingly, the loss of ICC is also found in gastroparesis with increased CD45 and CD68 immunoreactivity (14), indicating potential similarities between STC and gastroparesis. The diagnostic and prognostic roles of miRNAs in various types of malignancies and disorders have been explored over the last decade $(15,16)$. Previously, a negative correlation between miRNA-128 and macrophages in STC was reported by Liu et al (17). However, the exact mechanism underlying STC remains largely unknown.

Thus, the present study was conducted to characterize miRNA expression and identify key miRNAs associated with STC using weighted gene correlation network analysis (WGCNA). WGCNA was established to identify and delineate significant modules comprising highly clustered genes and to further correlate those genes modules with certain clinical traits $(18,19)$. Distinct from the differential gene expression analysis strategy, WGCNA upgrades and facilitates gene screening and biological applications/processes. This bioinformatics analysis was performed using the publicly available miRNA expression profile GSE57969.

\section{Materials and methods}

Gene expression profile in Gene Expression Omnibus (GEO). The non-coding RNA expression profile, GSE57969, was downloaded from the GEO database (http://www.ncbi.nlm. nih.gov/geo/) (20) with annotated clinical information. The GSE57969 dataset consisted of six colon tissues from six patients (five females and one male) with STC who underwent colectomy (total colectomy and ileorectal anastomosis or subtotal colectomy with antiperistaltic cecoproctostomy) and six normal colon tissues as controls from six patients (five females and one male) with colon cancer in a tumor-free area at least $5 \mathrm{~cm}$ away from the primary lesions. Notably, patients in the control group were free from constipation. The microarrays of all samples were scanned. The data were processed by Agilent Feature Extraction software version 9.5.3 and Gene Spring software version 11.0 (Agilent Technologies, Inc.) with normalization. The normalized data were annotated by the platform GPL11487 (Agilent-021827 Human miRNA Microarray) (17).

Identification of differentially expressed miRNAs. The differentially expressed miRNAs were determined by GEO2R (https://www.ncbi.nlm.nih.gov/geo/geo2r), a web-based analysis tool for in silico bioinformatics analysis (21). The cut-off value for the differentially expressed miRNAs was defined as an adjusted P-value of $<0.05$. The heatmap and volcano was generated by R software (3.6.0, https://www.r-project.org) (22).

WGCNA. WGCNA was performed in an R environment $(18,19)$. The entire standardized miRNA expression profile, GSE57696, was measured by Pearson's correlation coefficients. The generated similarity matrix was used to describe the concordance between miRNA expression. Afterwards, an adjacency matrix was generated by the similarity matrix according to the selected power adjacency function, predefined as the connection strengths on each node $(18,19)$. In the present study, a power $\beta=9$ was selected as the most qualified value based on the criterion of the scale-free topology (19).

Next, the topological overlap measurement (TOM; a dissimilarity measure of average linkage hierarchical clustering) was performed. Topological overlap can be measured to the interconnectedness of networks in terms of overlap (23). Within the dendrogram, modules are represented as tree branches with a dynamic tree-cut algorithm using hybrid branch cutting method $(23,24)$. To further quantify the associations between modules and clinical traits (gender and disease status, STC or control), the module eigengenes were calculated and correlated with the clinical traits (Module-Trait Relationships) $(19,24)$.

The association between individual genes and STC was defined by the gene significance (GS) (19). Module membership (also known as eigengene-based connectivity) was defined as the correlation between the module eigengene and the gene expression profile, with the aim to quantify the similarity of every module to the matrix $(18,19)$. The module with the most significant P-value and correlation was chosen for further processing. The miRNAs with a correlation value $>0.6$ or $<-0.6$ for STC were identified as the key miRNAs.

Functional enrichment of the key miRNA target genes. The miRNA target genes were predicted by three independent web-based databases for miRNA target prediction, TargetScanHuman (http://targetscan.org/vert_72/) (25), miRDB (http://mirdb.org/) (26) and microT-CDS (http://diana. imis.athena-innovation.gr/DianaTools/index.php?r=MicroT_ CDS/index) (27). All the predicted miRNA targets were subsequently annotated by Kyoto Encyclopedia of Genes and Genomes (KEGG) and Gene Ontology (GO), which breaks processes down into Biological Process (BP), Cellular Components (CC) and Molecular Functions (MF) with the Database for Annotation, Visualization and Integrated Discovery (https://david.ncifcrf.gov/) (28-30). Additional pathway enrichment was performed using the BioCarta database, a newly established dataset collection for mining genes (31).

Protein-Protein Interaction (PPI) networks of key miRNA target genes. The PPI networks of the miRNA target genes were constructed by the Search Tool for the Retrieval of Interacting Genes database and further visualized by the Cytoscape program (version 3.6.0) $(32,33)$. The cut-off values were: Degree $\geq 1$, node score $=0.2$ and k-score $=2$ with the max depth $=100$. The top-scoring modules were identified by the Molecular Complex Detection (MCODE) algorithm (34). The MCODE was embedded in Cytoscape software. Each top-scoring module was separately displayed in PPI as well. Moreover, the top 10 genes with the highest degrees were identified as the hub genes.

Statistical analysis. R software (3.6.0, https://www.r-project. org) (22) and GraphPad Prism 5.0 software (GraphPad Software, Inc.) were selected for illustration and statistical analysis. For correlation analysis, a Pearson test was used for measurement. Adjusted P-values were generated by the GEO2R web tool and used for statistical comparison in terms of the initial identification of differentially expressed genes. 
A

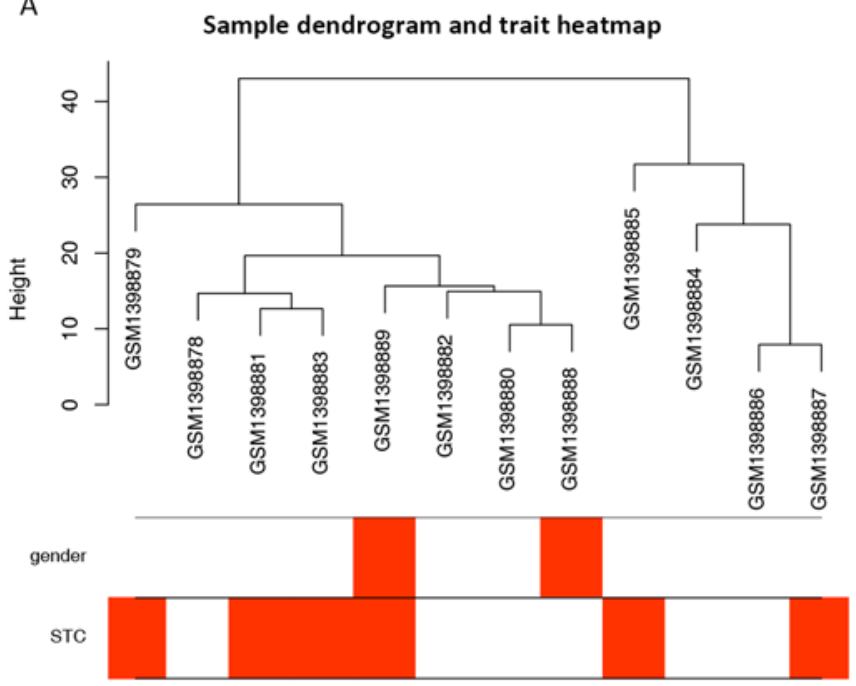

C

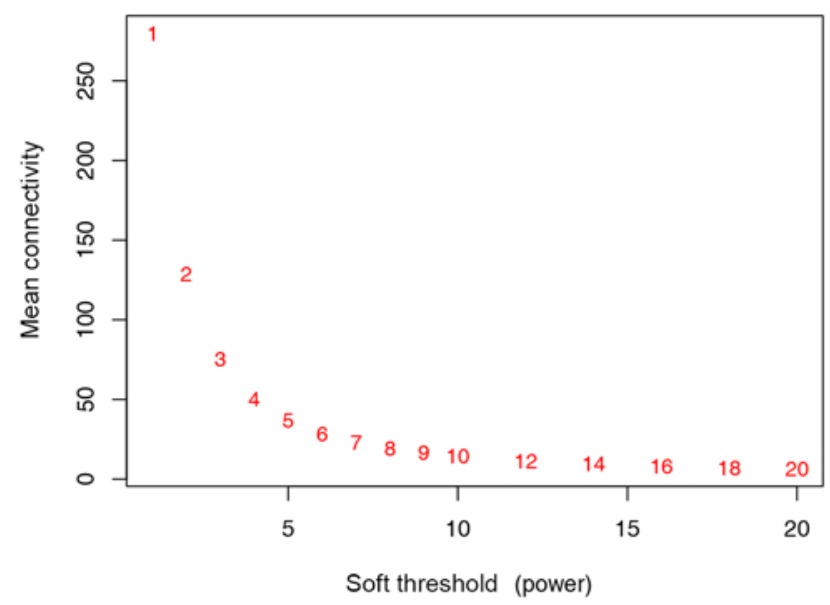

B

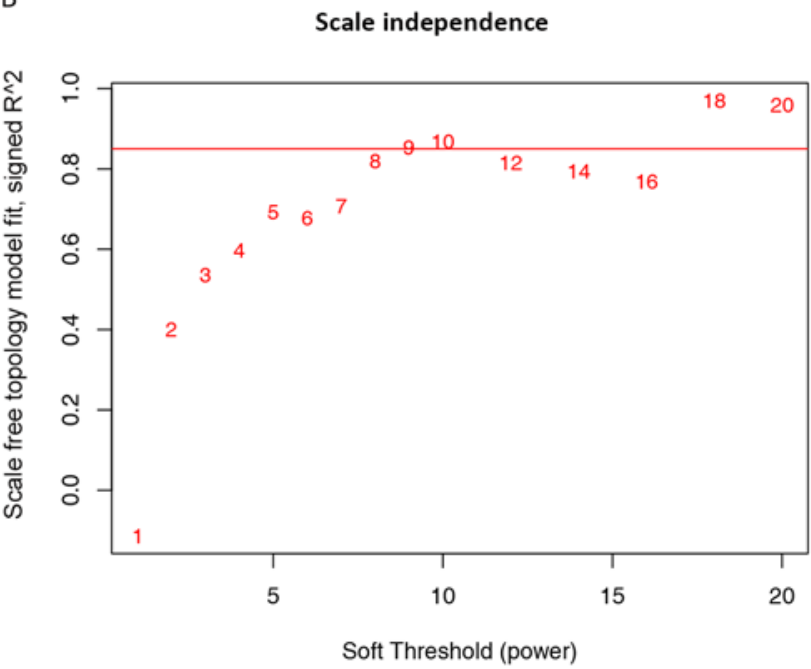

D

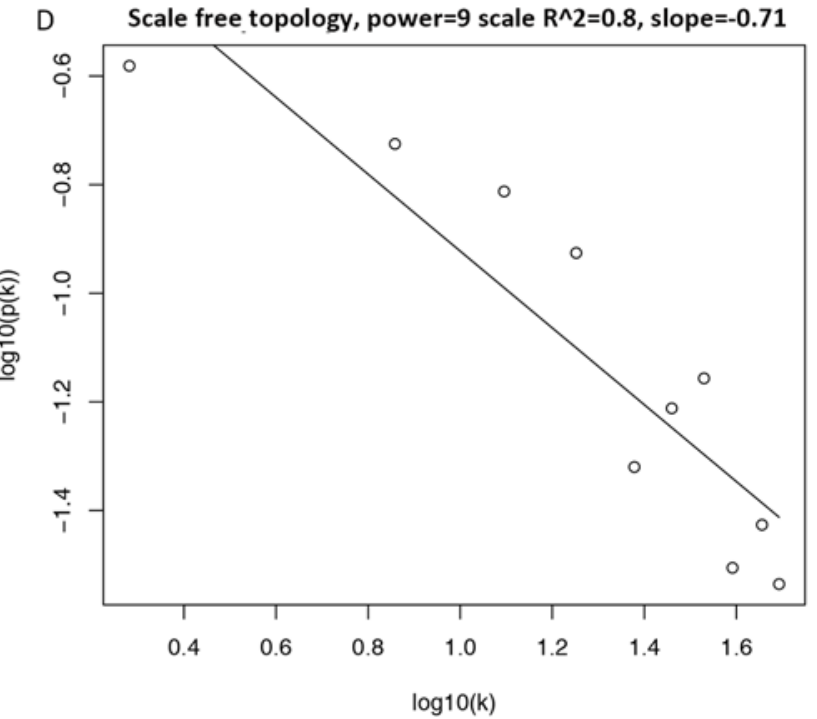

Figure 1. Cluster tree of STC samples and determination of soft-threshold power in weighted gene co-expression network analysis. (A) Cluster tree of STC samples; gender in red, male; gender in white, female; STC in red, STC samples; STC in white, control tissues. (B) Scale-free fit index for soft-threshold powers $\beta$. (C) Mean connectivity for each power. (D) Scale-free topology checking with $\beta=9$. STC, slow-transit constipation.

$\mathrm{P}<0.05$ was considered to indicate a statistically significant difference in terms of rest condition.

\section{Results}

WGCNA. The information of the included normalized samples for GSE57969 is displayed in Table SI and Fig. S1. The workflow of the bioinformatics analysis is displayed (Fig. S2). The differentially expressed miRNAs between the normal and STC groups were analyzed based on the GEO2R web tool. Interestingly, none of the miRNAs displayed remarkable differential expression between the normal and STC groups given all the adjusted P-values were $>0.05$ (Table SII). However, the heatmap and volcano plot of miRNAs with $\mathrm{P}<0.05$ were also exhibited (Figs. S3 and S4).

A total of 961 miRNAs and 12 samples retrieved from the original expression profiles were used for the co-expression network (Fig. 1A). A power of $\beta=9$ was selected as the optimal soft-threshold value for the subsequent construction of networks (Fig. 1B-D). The network was selected as unsigned with a minimal module size of 30 and a merge cut height of 0.25; however, a total of 12 clustered miRNAs, represented by colored modules were determined. The correlations between each module and clinical traits (gender and STC status) were calculated (Fig. 2A and B). Within the 12 modules, a TOM plot visualized the connectivity of the modules with color-coded depiction (all the colors were randomly assigned to each determined module; Fig. 2C). Although no module showed significant correlations with STC in clinical traits (Fig. 2B), the present study individually explored the GS and the module membership in each module (Fig. 3A-L). In fact, the module membership of the blue module showed the most significant correlation with GS (cor=0.87, p=2.8e-39), whereas the turquoise module was the second most significant (cor=-0.71, $\mathrm{p}=1.1 \mathrm{e}-31$ ). Moreover, seven key miRNAs were determined with five miRNAs from the turquoise module (hsa-miR-20b, hsa-miR-128, hsa-miR-129-3p, hsa-miR-30b and hsa-miR-340), one miRNA from the blue module (hsa-miR-619) and one from the black module (hsa-miR-486-3p) (Tables I and SIII). 


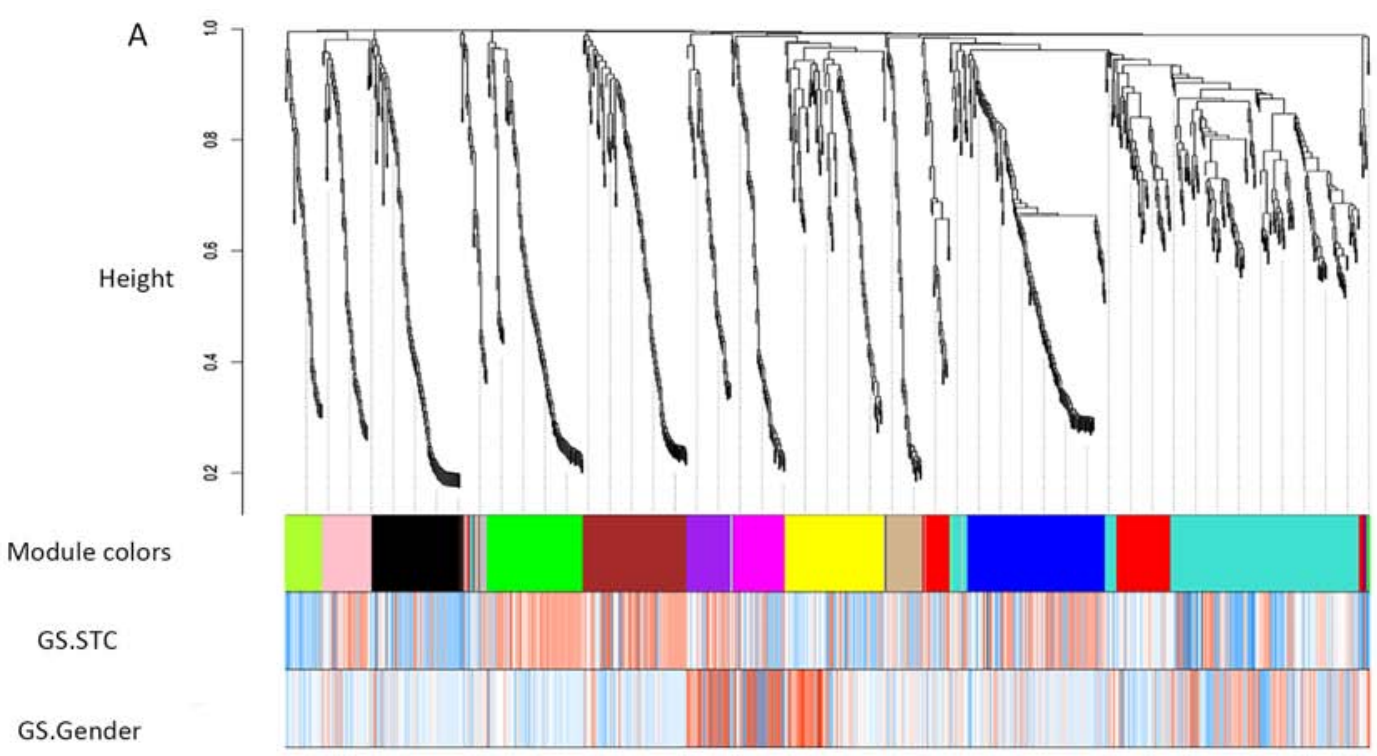

\section{B}

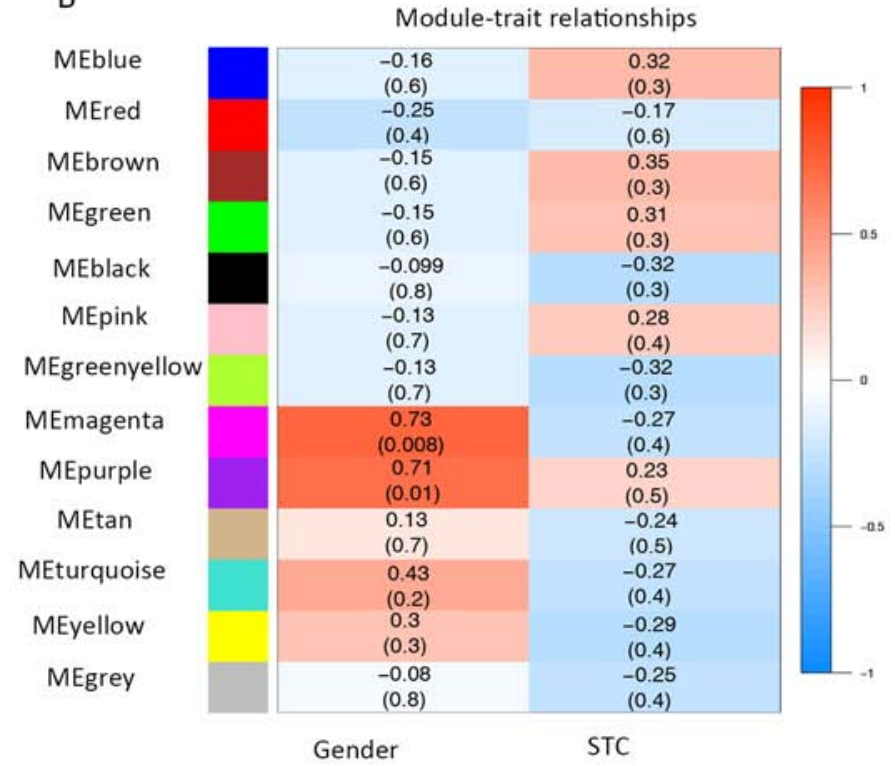

C

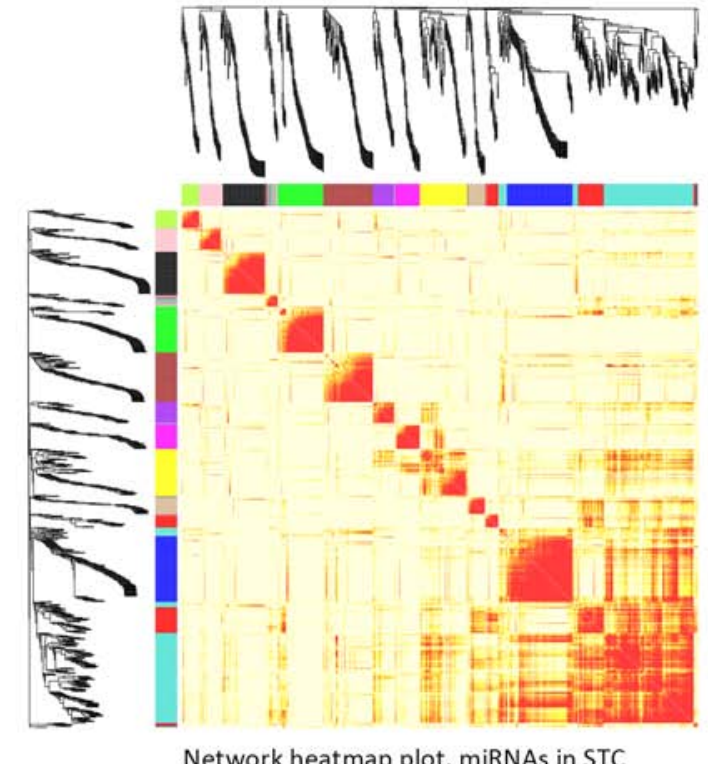

Figure 2. Hierarchical clustering dendrogram of the included modules with associated traits. (A) The color bands indicate various modules based on the dynamic hybrid branch cutting method. The upper color band shows the results of automatic single block analysis. The middle and bottom color bands show the GS (miRNA significance) in association with STC and gender. In the middle color band, red indicates a high positive correlation with STC, whereas blue indicates a high negative correlation with STC. In the lower band, red indicates a high positive correlation between gender and each miRNA while blue indicates a negative correlation. (B) Correlations (and P-values) between the modules (rows) identified by the single block analysis, and traits (columns); the most correlated colored module with STC was identified by this plot for further analysis. (C) A TOM plot of the network connections in miRNAs. The dark red color indicates high topological overlap, whereas the lighter yellow color represents low topological overlap. The cluster trees and colorful modules are shown at the left and top. TOM, Topological overlap matrix; STC, slow-transit constipation; GS, gene significance.

Functional enrichment of miRNA target genes. A total of 2,077 genes were predicted as targets of the key miRNAs. GO enrichment analysis indicated that the 'protein modification process' and 'cellular protein modification process' were the most significantly enriched BP, 'nucleoplasm' was the most enriched CC and 'enzyme binding' was the most enriched MF (Fig. 4). KEGG analysis revealed that the most significantly enriched term was the 'cAMP signalling pathway' (Fig. 5). In addition, pathway enrichment analysis from the BioCarta database was performed with no significantly enriched pathways (FDR $>0.05$ ) identified (Table SIV). The PPI networks were constructed with 641 nodes and 2,414 edges (Fig. 6). The hub genes included calmodulin (CALM)2, CALM1, histone deacetylase (HDAC)3, glycogen synthase kinase $3 \beta$, HDAC9, heat-shock protein family A member 8 , G-protein subunit $\gamma(\mathrm{GNG}) 13$, HECT domain and ankyrin repeat containing E3 ubiquitin protein ligase 1, GNG10 and GNG7 (Tables II and SV). The top-scoring modules identified in the PPI are displayed with the most significantly enriched KEGG pathways. Top-scoring modules identified in the Protein-Protein Interaction network using the MCODE algorithm (Figs. 7A, 8A and 9A). The first module was showed in PPI network (Fig. 7A). 'Morphine addiction', 'glutamatergic synapse' and the 'chemokine signalling pathway' were the 
Table I. Key miRNAs identified in association with STC based on the GS and MM.

\begin{tabular}{|c|c|c|c|c|}
\hline ID & Module & Cor.STC & By GEO2R (P-value) & By Liu et al (17) (P-value) \\
\hline hsa-miR-619 & Blue & 0.691847 & - & - \\
\hline hsa-miR-20b & Turquoise & -0.60402 & 0.041 & 0.041 \\
\hline hsa-miR-128 & Turquoise & -0.61819 & 0.0479 & 0.035 \\
\hline hsa-miR-486-3p & Black & -0.64059 & - & - \\
\hline hsa-miR-129-3p & Turquoise & -0.64954 & 0.0186 & 0.023 \\
\hline hsa-miR-30b & Tturquoise & -0.69318 & 0.0103 & 0.015 \\
\hline hsa-miR-340 & Turquoise & -0.70344 & 0.0107 & - \\
\hline
\end{tabular}

The key miRNAs were also compared with the results from GEO2R and Liu et al (17). Cor, correlation; GS, gene significance; MM, module membership; miR/miRNA, microRNA; STC, slow-transit constipation.

A
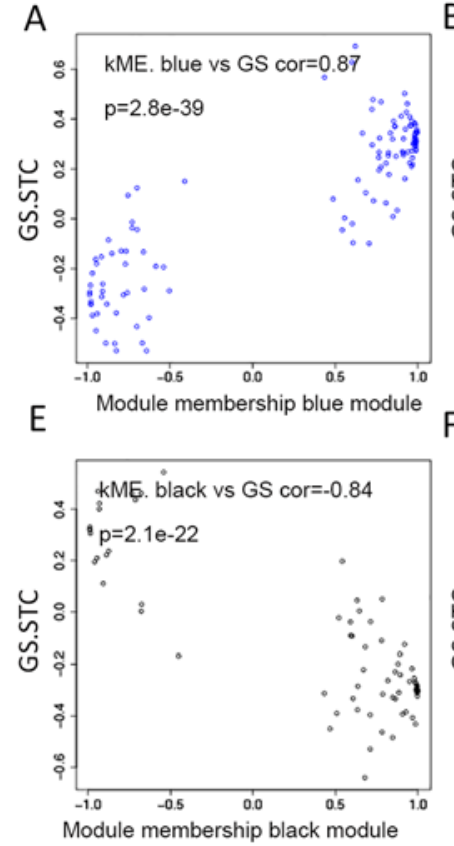
I

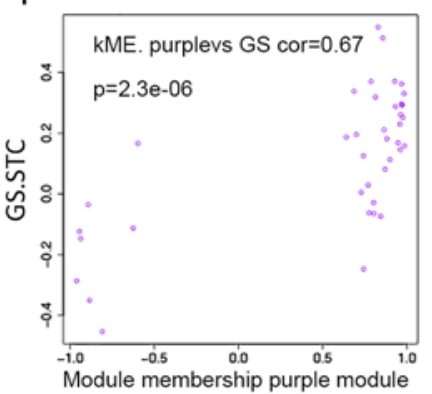

B

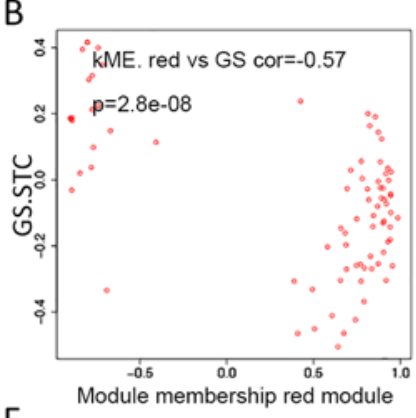

$F$

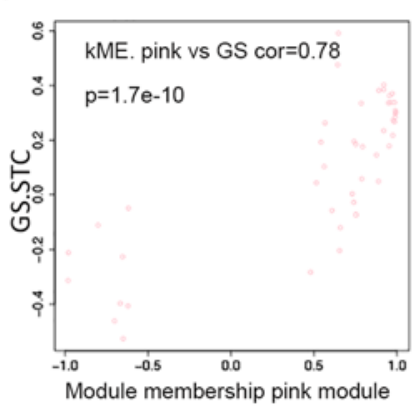

J

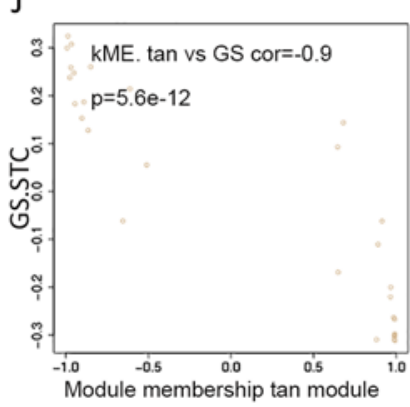

C

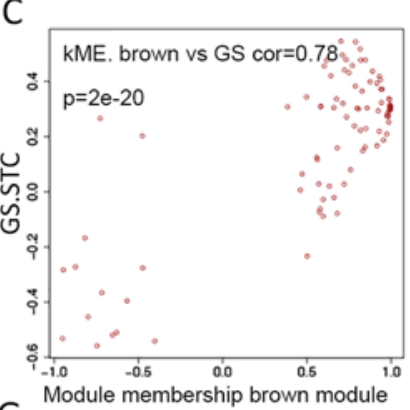

G

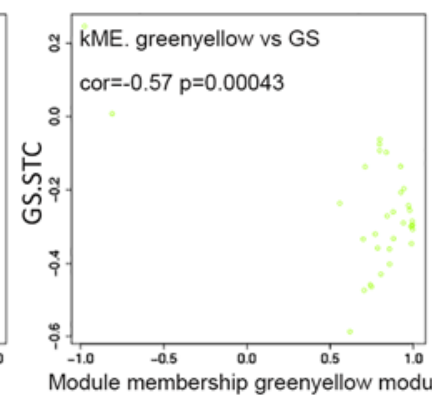

$\mathrm{K}$

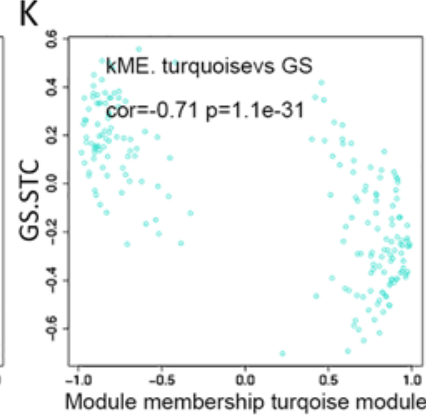

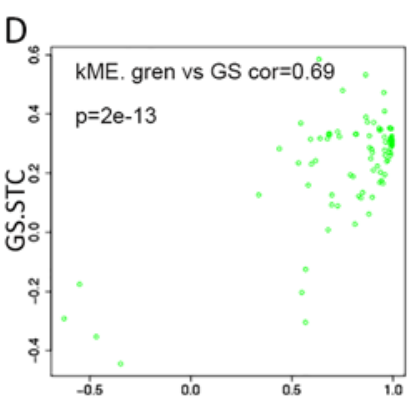

$\mathrm{H}$

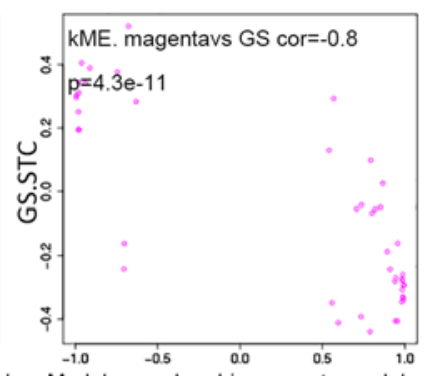

$\mathrm{L}$

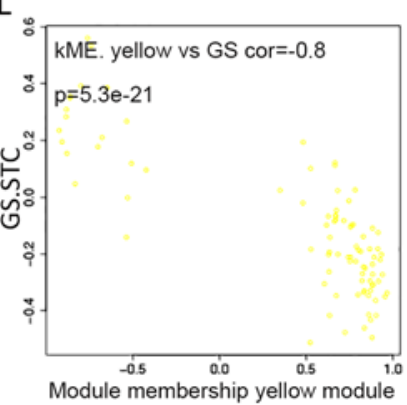

Figure 3. Correlation between the GS. STC and the kME in each colored module. (A) kME blue vs. GS. (B) kME red vs. GS. (C) kME brown vs. GS. (D) kME green vs. GS. (E) kME black vs. GS. (F) kME pink vs. GS. (G) kME green yellow vs. GS. (H) kME magenta vs. GS. (I) kME purple vs. GS. (J) kME tan vs. GS. (K) kME turquoise vs. GS. (L) kME yellow vs. GS. Dots represent one gene. The scatterplot results were analyzed by a Pearson correlation analysis. GS, gene significance; kME, module membership; GS.STC, gene significance in slow-transit constipation.

most significantly enriched pathways in module 1 (the module with highest score; Fig. 7B). 'Spliceosome' was the only significantly enriched KEGG pathway in module 2 (the module with the second highest score; Fig. 8B). 'Alcoholism' was the only significantly enriched pathway in module 3 (the module with the third highest score; Fig. 9B). In addition, topological features were also characterized (Tables SVI and SVII). Specifically, the top 20 genes with highest degree were listed, along with average shortest path length, betweenness centrality, closeness centrality, clustering coefficient, neighborhood connectivity, radiality and topological coefficient parameters. The networks identified by MCODE were also characterized with simpler 


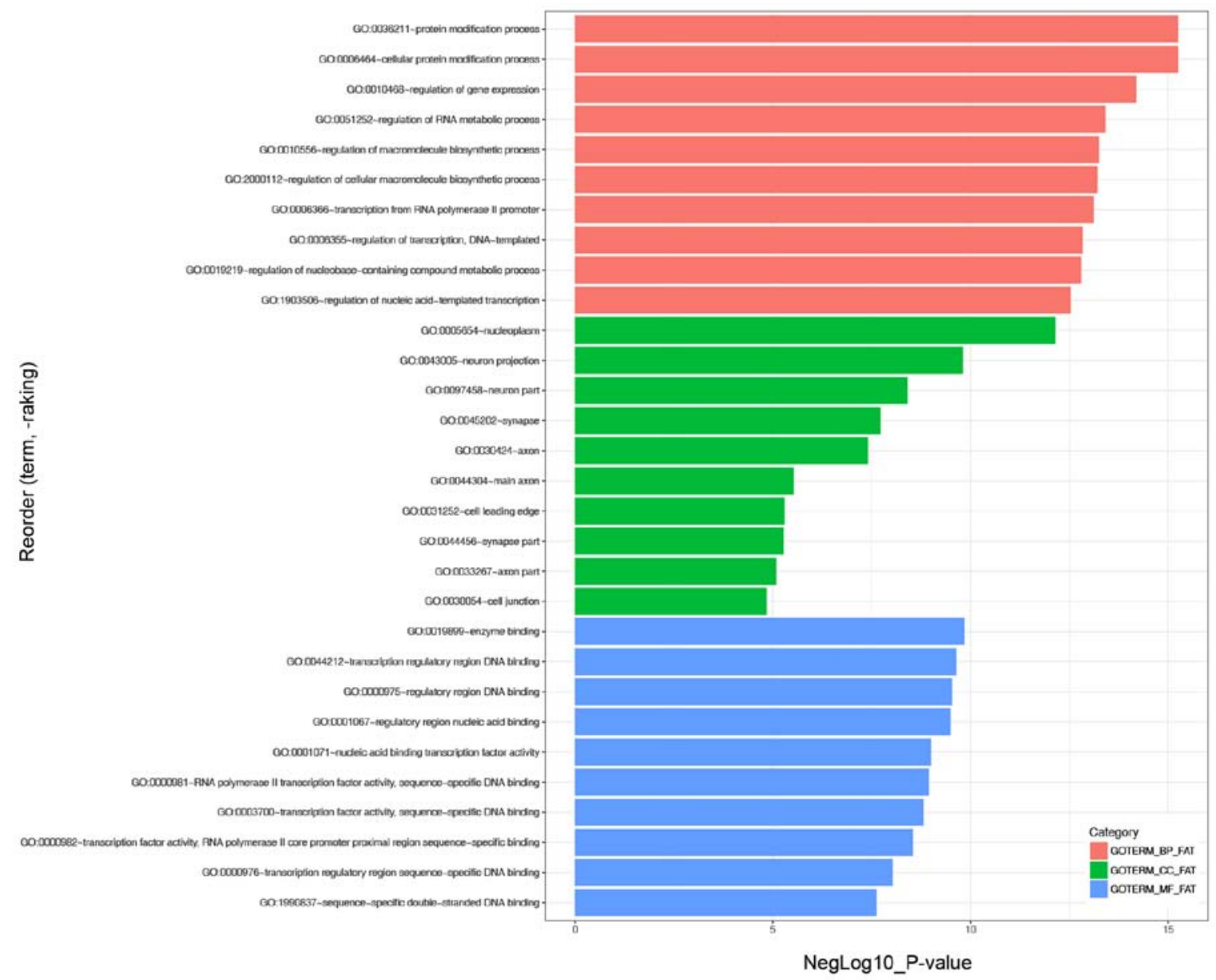

Figure 4. Functional enrichment results of all key miRNA target genes. GO results of the included genes (predicted target genes of key miRNAs); red, BP in GO; green, CC in GO; blue, MF in GO; the P-value was visualized by - $\log 10$ (P-value). The GO results were statistically analyzed by the Database for Annotation, Visualization and Integrated Discovery (https://david.ncifcrf.gov/). GO, Gene Ontology; BP, Biological Process; CC, Cellular Components; MF, Molecular Functions.

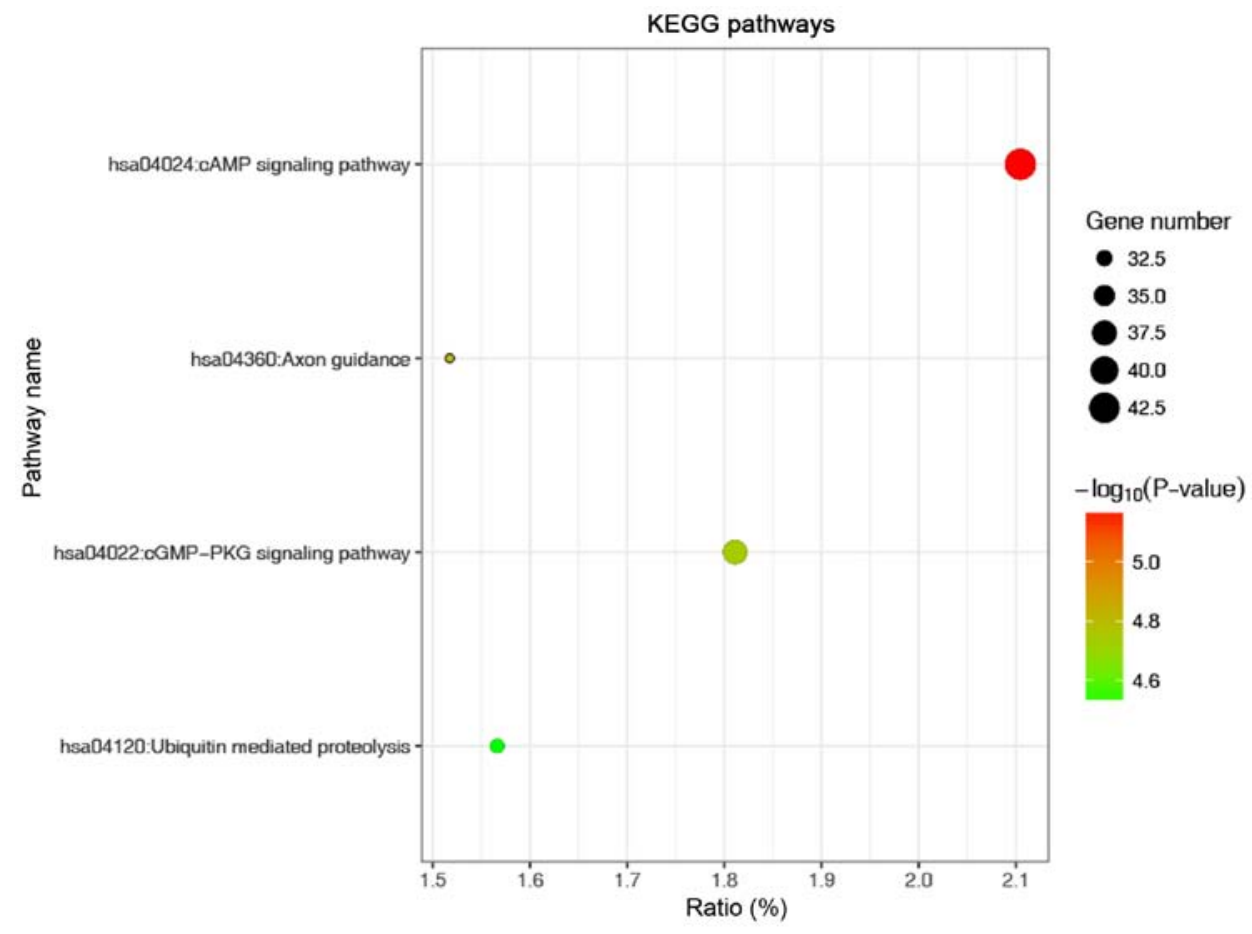

Figure 5. KEGG results of the included genes. The P-value was visualized by -log10 (P-value) from red to green. Red indicates greater significance. The dot size was in proportion to the enriched gene number in each pathway. The KEGG results were statistically analyzed by the Database for Annotation, Visualization and Integrated Discovery (https://david.ncifcrf.gov/). KEGG, Kyoto Encyclopedia of Genes and Genomes. 
Table II. Hub genes identified in the Protein-Protein Interaction networks.

\begin{tabular}{lll}
\hline Gene symbol & Degree & \\
\hline CALM2 & 55 & calmodulin 2 \\
CALM1 & 52 & calmodulin 1 \\
HDAC3 & 42 & histone deacetylase 3 \\
GSK3B & 41 & glycogen synthase kinase $3 \beta$ \\
HDAC9 & 41 & histone deacetylase 9 \\
HSPA8 & 41 & heat shock protein family A member 8 \\
GNG13 & 40 & G-protein subunit $\gamma$ 13 \\
HACE1 & 39 & G-protein subunit $\gamma 10$ \\
GNG10 & 39 & G-protein subunit $\gamma 7$ \\
GNG7 & 38 &
\end{tabular}

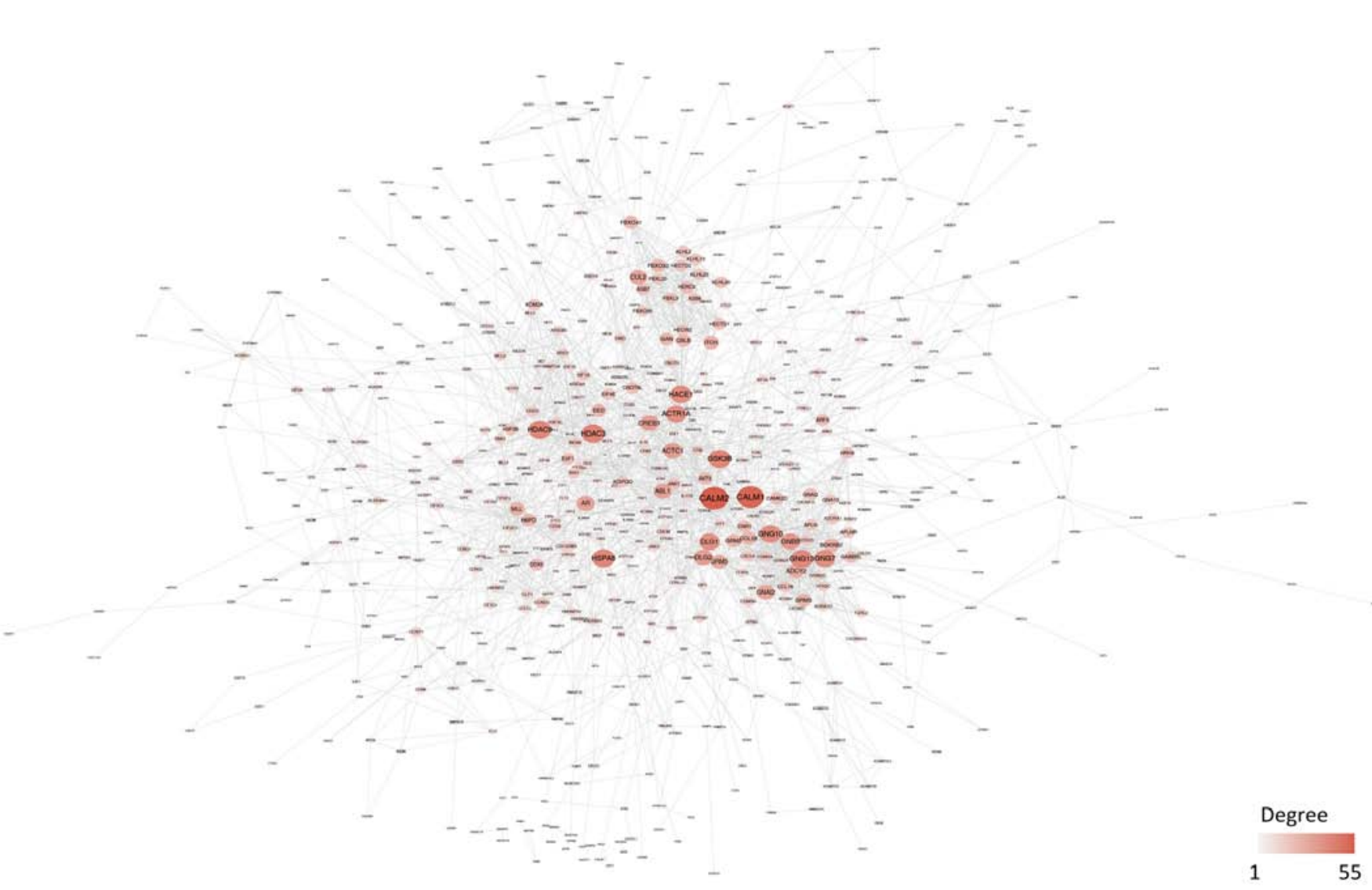

Figure 6. Protein-Protein Interaction networks of key microRNA target genes. The size and color of each dot is reflected by the degree value. The hub genes with highest degrees displayed the largest size and darkest color.

parameters, including MCODE scores, nodes, edges and genes IDs.

\section{Discussion}

To the best of the authors knowledge, this is the first WGCNA-based bioinformatics study for an STC-associated miRNA expression profile (GSE57969) in the GEO platform. This study provided unique insights and meaningful evidence of potential miRNAs, genes and pathways involved in the pathogenesis of STC. The GEO platform was utilized for these studies, which has the only gene expression profile and
miRNA expression profile on STC. Previous bioinformatics studies related to STC currently remain limited. Specifically, Liu et al (17) discovered a significant negative correlation between miRNA-128, and colonic macrophage numbers during the pathogenesis of STC. Other studies that have focused on STC were primarily associated with clinical practice, with sparse investigation into genomic expression profiles (2-5). In summary, this is the first study on the bioinformatic analysis of miRNA expression profile in STC. Of note, the original article on GSE57969 showed that miR-128 is significantly decreased in STC samples compared with the control samples in GSE57969. 
A

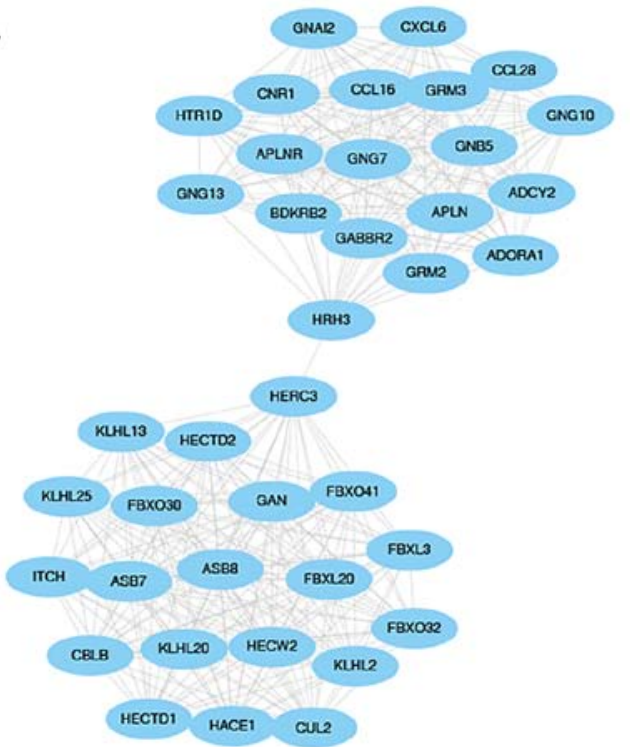

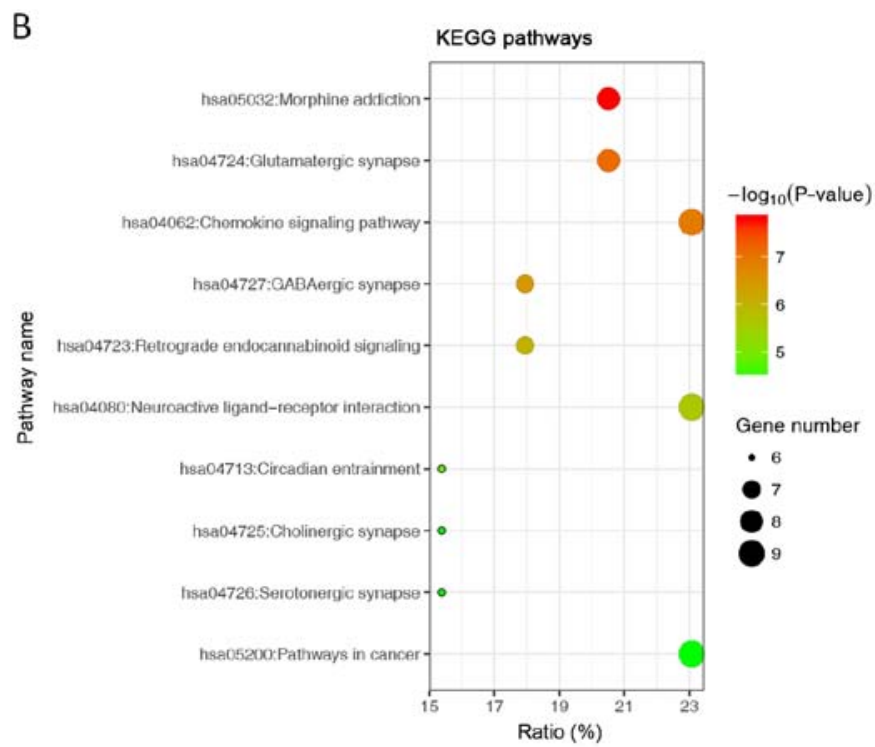

Figure 7. Identification of top highest-scored module related to the PPI networks using MCODE. (A) Top-scoring module identified in the Protein-Protein Interaction network using the MCODE algorithm, this network was determined by MCODE, embedded in Cytoscape software; (B) corresponding KEGG pathway results. All the scoring results were output by MCODE algorithm. MCODE, Molecular Complex Detection; KEGG, Kyoto Encyclopedia of Genes and Genomes.

A

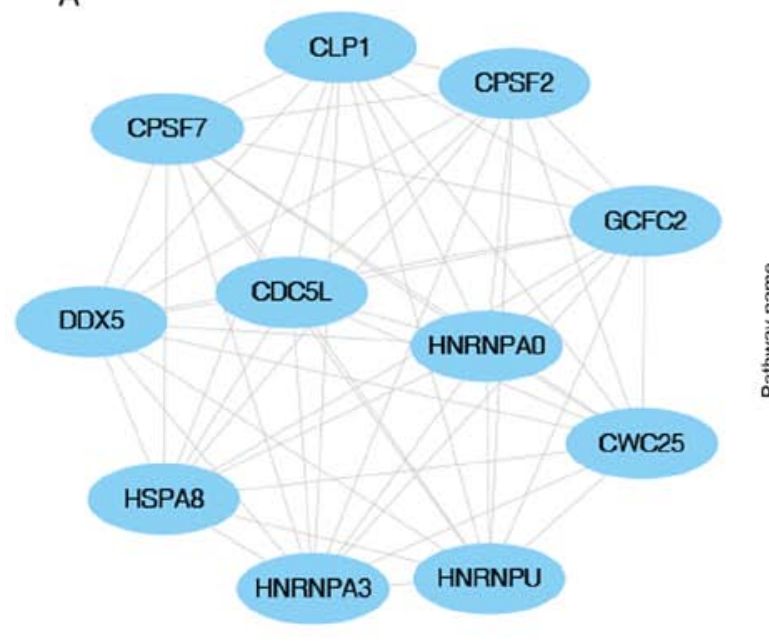

B

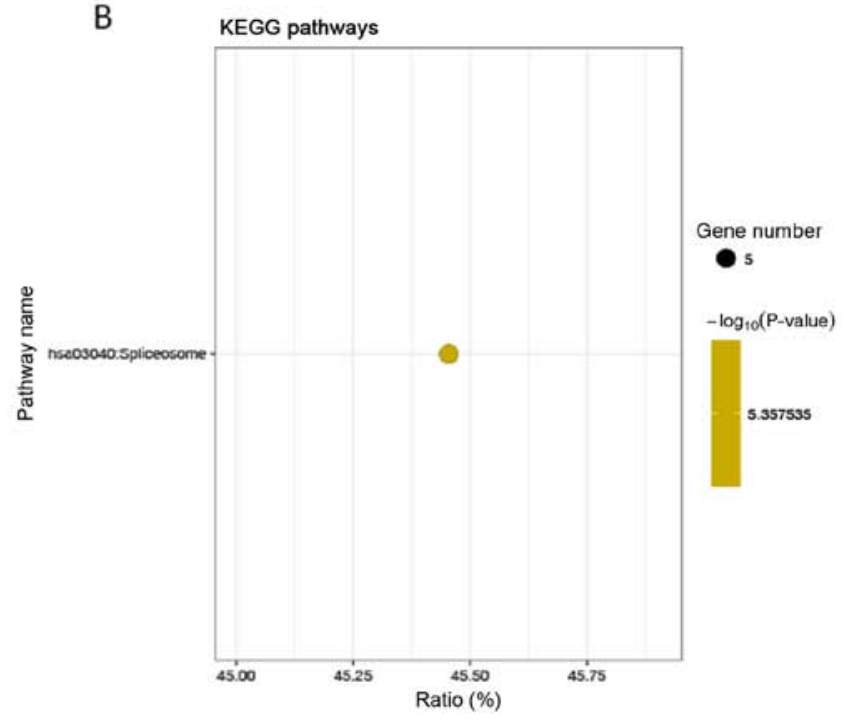

Figure 8. Identification of second highest-scored module related to the PPI networks using MCODE algorithm. (A) Second highest scoring module identified in the Protein-Protein Interaction network using the MCODE algorithm and (B) corresponding KEGG pathway result. All the scoring results were output by MCODE algorithm. MCODE, Molecular Complex Detection; KEGG, Kyoto Encyclopedia of Genes and Genomes.

The present study reanalyzed the expression profile with WGCNA and other bioinformatics strategies, and rediscovered additional key miRNAs and pathways underlying the pathogenesis of STC. Consistently, miR-128 was found to be a key miRNA in the present results, corroborating what was found by Liu et al (17). Furthermore, the present study identified a correlation between hsa-miR-619 (positive correlation), 20b (negative correlation), 486-3p (negative correlation), 129-3p (negative correlation), 30b (negative correlation), 340 (negative correlation) and STC by WGCNA (Table I). Interestingly, only hsa-miR-619 featured a positive correlation with the presence of STC (cor=0.692), whereas the remaining six key miRNAs showed a negative correlation with the presence of STC.
Previous studies have demonstrated that both the ganglionic density and size, and the number of ICCs were reduced in patients with STC and megacolon (11-13,35). More molecular biomarkers, instead of mere histopathological features, were suggested to identify STC. Currently, surgical interventions for STC remain largely debatable. A previous study has reported that diverse postoperative complications and functional anomalies may limit postoperative satisfaction in patients with STC (36). Nonetheless, biofeedback, a recommended clinical management for chronic constipation, is less effective in patients with STC than in patients with pelvic floor dyssynergia (37). These findings reflect the clinical complexity and heterogeneity of STC. Notably, the involvement of miRNAs 
A

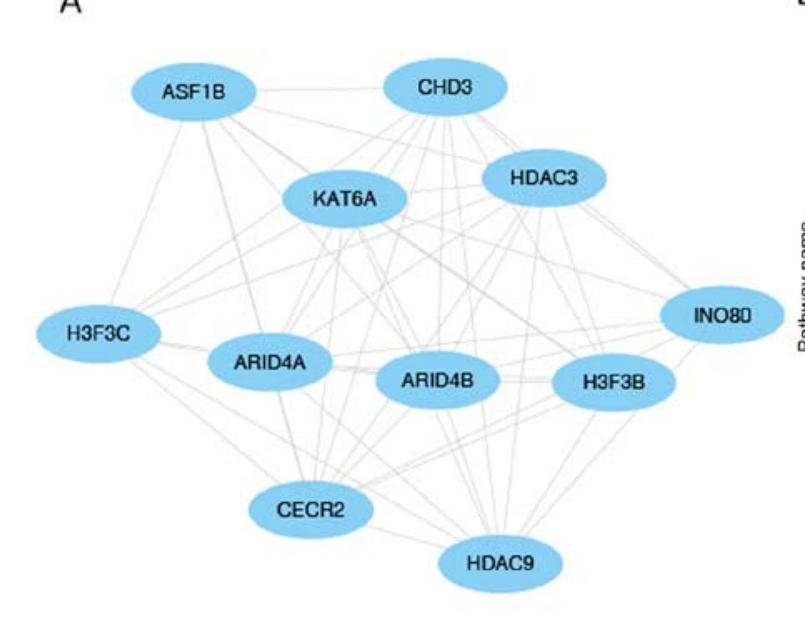

B

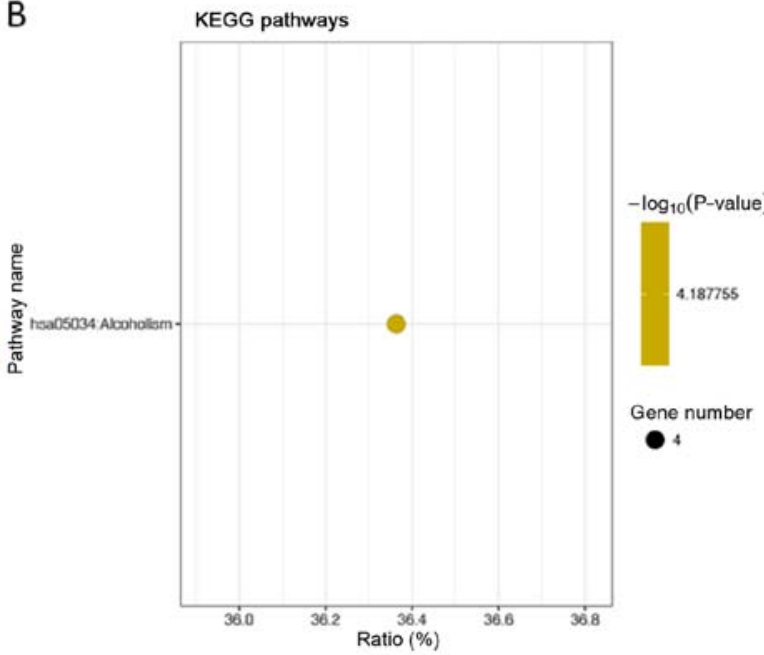

Figure 9. Identification of third highest-scored module related to the PPI networks using MCODE algorithm (A) Third highest scoring module identified in the Protein-Protein Interaction network using the MCODE algorithm and (B) corresponding KEGG pathway result. All the scoring results were output by MCODE algorithm. MCODE, molecular Complex Detection; KEGG, Kyoto Encyclopedia of Genes and Genomes.

could provide insight into the pathogenesis and therapeutic options for STC.

Generally, in WGCNA, correlated gene expression profiles indicate a potential collaboration or pathway co-involvement, which contributes to the correlation of certain clinical phenotypes. All genes were weighted with the connection strengths and marked by a power function $(18,19)$. All the miRNAs were clustered into 12 subsets, represented by colored modules. Next, each module was linked to clinical information for further target gene annotations.

CALM1 and CALM2 were identified as the hub genes in the present study. The CALM family is a commonly studied group of $\mathrm{Ca}^{2+}$-sensing proteins, particularly in relation to secretory diarrhoea $(38-40)$. CALM proteins display numerous $\mathrm{Ca}^{2+}$-dependent biological functions by directing a variety of target-specific activations (38). It is of note that the cAMP signalling identified in the KEGG pathway is also closely associated with the CALM family in terms of pro-arrhythmic $\mathrm{Ca}^{2+}$ waves (41). Notably, one of the central factors in intestinal motility, ICC, has been found to be highly correlated with $\mathrm{Ca}^{2+}$ in terms of the initiation of pacemaker activity $(13,42,43)$. In fact, ICC exhibits a specialized, secondary voltage-independent slow wave current yet to be elucidated. Some studies have reported that this current is activated by a $\mathrm{Cl}^{-}$selective conductance (44). The redistribution of extracellular $\mathrm{Ca}^{2+}$ or replacement of $\mathrm{Ca}^{2+}$ could influence the rhythmic waves (44). In fact, the present results indicated the possible roles of the CALM family and the involvement of the cAMP signalling pathway in the potential underlying mechanism of STC.

In addition, different results between KEGG annotation and BioCarta were possibly due to the different referential background and embedded algorithm. However, as this is based on in silico analysis, further experimental validation is needed to confirm these conclusions. Another limitation of the present study is the relatively small sample size, which may be confounded by heterogeneous factors. Given the limited gene expression resources of the miRNAs of STC in GEO, a global picture of the miRNA regulatory mechanisms awaits further consolidation. Undeniably, the minimal recommended sample size of WGCNA is 15 (19), whereas only 12 samples were analyzed in this study. However, this unique miRNA microarray enabled the first WGCNA-based bioinformatics analysis of STC. Moreover, clinically resected sample sizes of STC are generally limited in China, partially because STC has not been fully recognized by patients who prefer treatment without surgery. Thus, STC resected specimens are relatively hard to get and the underlying pathogenesis remains largely unclear. In the present study, no reverse transcription-quantitative PCR was performed to validate the results. It may take at least 3-5 years for a comparable STC sample size to be attained.

\section{Acknowledgements}

The authors would like to thank Dr Ernest Johann Helwig (Tongji Medical College of Huazhong University of Science and Technology) for his insightful discussions and revision of the manuscript.

\section{Funding}

This study was supported by the National Natural Science Foundation of China (grant nos. 81402423, 81572818 and 81871984), and the Shanghai Municipal Commission of Health and Family Planning (grant no. 2017YQ062).

\section{Availability of data and materials}

All data generated or analyzed during this study are included in this published article.

\section{Authors' contributions}

CY, LuZ, BF, LuyangZ and PX carried out data analysis. CY, LuZ, JM, MZ, FD and JS drafted the manuscript. CY, JS, $\mathrm{JM}$ and $\mathrm{MZ}$ participated in study design and data collection. 
CY, BF and FD contributed substantially to the refinement of the conception and design of this study and were major contributor to the revision of the manuscript. All authors read and approved the final manuscript.

\section{Ethics approval and consent to participate}

Not applicable.

\section{Patient consent for publication}

Not applicable.

\section{Competing interests}

The authors declare that they have no competing interests.

\section{References}

1. Pare P, Ferrazzi S, Thompson WG, Irvine EJ and Rance L: An epidemiological survey of constipation in Canada: Definitions, rates, demographics, and predictors of health care seeking. Am J Gastroenterol 96: 3130-3137, 2001.

2. Lembo $A$ and Camilleri M: Chronic constipation. N Engl J Med 349: 1360-1368, 2003.

3. Sonnenberg A and Koch TR: Physician visits in the United States for constipation: 1958 to 1986. Dig Dis Sci 34: 606-611, 1989.

4. Heaton KW, Radvan J, Cripps H, Mountford RA, Braddon FE and Hughes AO: Defecation frequency and timing, and stool form in the general population: A prospective study. Gut 33: 818-824, 1992 .

5. Preston DM and Lennard-Jones JE: Severe chronic constipation of young women: 'Idiopathic slow transit constipation'. Gut 27: 41-48, 1986.

6. Rao SS and Go JT: Update on the management of constipation in the elderly: New treatment options. Clin Interv Aging 5: 163-171, 2010.

7. Portalatin $\mathrm{M}$ and Winstead $\mathrm{N}$ : Medical management of constipation. Clin Colon Rectal Surg 25: 12-19, 2012.

8. Karlbom U, Påhlman L, Nilsson S and Graf W: Relationships between defecographic findings, rectal emptying, and colonic transit time in constipated patients. Gut 36: 907-912, 1995.

9. Prather CM: Subtypes of constipation: Sorting out the confusion. Rev Gastroenterol Disord 4 (Suppl 2): S11-S16, 2004.

10. Mertz H, Naliboff B and Mayer E: Physiology of refractory chronic constipation. Am J Gastroenterol 94: 609-615, 1999.

11. Tzavella K, Riepl RL, Klauser AG, Voderholzer WA, Schindlbeck NE and Müller-Lissner SA: Decreased substance $\mathrm{P}$ levels in rectal biopsies from patients with slow transit constipation. Eur J Gastroenterol Hepatol 8: 1207-1211, 1996.

12. Cortesini $C$, Cianchi F, Infantino A and Lise M: Nitric oxide synthase and VIP distribution in enteric nervous system in idiopathic chronic constipation. Dig Dis Sci 40: 2450-2455, 1995.

13. He CL, Burgart L, Wang L, Pemberton J, Young-Fadok T, Szurszewski J and Farrugia G: Decreased interstitial cell of Cajal volume in patients with slow-transit constipation. Gastroenterology 118: 14-21, 2000.

14. Grover M, Farrugia G, Lurken MS, Bernard CE, Faussone-Pellegrini MS, Smyrk TC, Parkman HP, Abell TL, Snape WJ, Hasler WL, et al: Cellular changes in diabetic and idiopathic gastroparesis. Gastroenterology 140: 1575-1585.e8, 2011.

15. Yanaihara N, Caplen N, Bowman E, Seike M, Kumamoto K, Yi M, Stephens RM, Okamoto A, Yokota J, Tanaka T, et al: Unique microRNA molecular profiles in lung cancer diagnosis and prognosis. Cancer Cell 9: 189-198, 2006.

16. Ferracin M, Veronese A and Negrini M: Micromarkers: miRNAs in cancer diagnosis and prognosis. Expert Rev Mol Diagn 10: 297-308, 2010.

17. Liu W, Zhang Q, Li S, Li L, Ding Z, Qian Q, Fan L and Jiang C: The relationship between colonic macrophages and microRNA-128 in the pathogenesis of slow transit constipation. Dig Dis Sci 60: 2304-2315, 2015.
18. Zhang B and Horvath S: A general framework for weighted gene co-expression network analysis. Stat Appl Genet Mol Biol 4: Article17, 2005.

19. Langfelder $P$ and Horvath S: WGCNA: An R package for weighted correlation network analysis. BMC Bioinformatics 9: 559, 2008.

20. Edgar R, Domrachev M and Lash AE: Gene expression omnibus: NCBI gene expression and hybridization array data repository. Nucleic Acids Res 30: 207-210, 2002.

21. Davis S and Meltzer PS: GEOquery: A bridge between the Gene Expression Omnibus (GEO) and BioConductor. Bioinformatics 23: 1846-1847, 2007.

22. R Core Team. R: A language and environment for statistical computing. R Foundation for Statistical Computing, Vienna, Austria, 2017. URL www.r-project.org. Accessed June 24, 2019.

23. Ravasz E, Somera AL, Mongru DA, Oltvai ZN and Barabási AL: Hierarchical organization of modularity in metabolic networks. Science 297: 1551-1555, 2002.

24. Langfelder P, Zhang B and Horvath S: Defining clusters from a hierarchical cluster tree: The dynamic tree cut package for $\mathrm{R}$. Bioinformatics 24: 719-720, 2007.

25. Agarwal V, Bell GW, Nam JW and Bartel DP: Predicting effective microRNA target sites in mammalian mRNAs. Elife 4: e05005, 2015.

26. Wang $\mathrm{X}$ : Improving microRNA target prediction by modeling with unambiguously identified microRNA-target pairs from CLIP-Ligation studies. Bioinformatics 32: 1316-1322, 2016.

27. Paraskevopoulou MD, Georgakilas G, Kostoulas N, Vlachos IS, Vergoulis T, Reczko M, Filippidis C, Dalamagas T and Hatzigeorgiou AG: DIANA-microT web server v5.0: Service integration into miRNA functional analysis workflows. Nucleic Acids Res 41: W169-W173, 2013.

28. Ashburner M, Ball CA, Blake JA, Botstein D, Butler H, Cherry JM, Davis AP, Dolinski K, Dwight SS, Eppig JT, et al: Gene Ontology: Tool for the unification of biology. Nat Genet 25: 25-29, 2000.

29. Kanehisa M and Goto S: KEGG: Kyoto encyclopedia of genes and genomes. Nucleic Acids Res 28: 27-30, 2000.

30. Huang DW, Sherman BT and Lempicki RA: Systematic and integrative analysis of large gene lists using DAVID bioinformatics resources. Nature Protoc 4: 44-57, 2009.

31. Rouillard AD, Gundersen GW, Fernandez NF, Wang Z, Monteiro CD, McDermott MG and Ma'ayan A: The harmonizome: A collection of processed datasets gathered to serve and mine knowledge about genes and proteins. Database (Oxford) 2016: baw100, 2016.

32. Szklarczyk D, Franceschini A, Wyder S, Forslund K, Heller D, Huerta-Cepas J, Simonovic M, Roth A, Santos A, Tsafou KP and Kuhn M: STRING v10: Protein-protein interaction networks, integrated over the tree of life. Nucleic Acids Res 43: D447-D452, 2014.

33. Shannon P, Markiel A, Ozier O, Baliga NS, Wang JT, Ramage D, Amin N, Schwikowski B and Ideker T: Cytoscape: A software environment for integrated models of biomolecular interaction networks. Genome Res 13: 2498-2504, 2003.

34. Bader GD and Hogue CW: An automated method for finding molecular complexes in large protein interaction networks. BMC Bioinformatics 4: 2, 2003.

35. Wedel T, Spiegler J, Soellner S, Roblick UJ, Schiedeck TH, Bruch HP and Krammer HJ: Enteric nerves and interstitial cells of Cajal are altered in patients with slow-transit constipation and megacolon. Gastroenterology 123: 1459-1467, 2002 .

36. Knowles CH, Scott M and Lunniss PJ: Outcome of colectomy for slow transit constipation. Ann Surg 230: 627, 1999.

37. Chiarioni G, Salandini L and Whitehead WE: Biofeedback benefits only patients with outlet dysfunction, not patients with isolated slow transit constipation. Gastroenterology 129: 86-97, 2005.

38. Chin D and Means AR: Calmodulin: A prototypical calcium sensor. Trends Cell Biol 10: 322-328, 2000.

39. Zavecz JH, Jackson TE, Limp GL and Yellin TO: Relationship between anti-diarrheal activity and binding to calmodulin. Eur J Pharmacol 78: 375-377, 1982.

40. Deng Y, Han X, Tang S, Xiao W, Tan Z, Zhou C, Wang M and Kang J: Magnolol and honokiol regulate the calcium-activated potassium channels signaling pathway in Enterotoxigenic Escherichia coli-induced diarrhea mice. Eur J Pharmacol 755: 66-73, 2015. 
41. Bobin P, Varin A, Lefebvre F, Fischmeister R, Vandecasteele G and Leroy $\mathrm{J}$ : Calmodulin kinase II inhibition limits the pro-arrhythmic $\mathrm{Ca}^{2+}$ waves induced by cAMP-phosphodiesterase inhibitors. Cardiovascular Res 110: 151-161, 2016.

42. Ward SM, Ördög T, Koh SD, Baker SA, Jun JY, Amberg G, Monaghan K and Sanders KM: Pacemaking in interstitial cells of Cajal depends upon calcium handling by endoplasmic reticulum and mitochondria. J Physiol 525: 355-361, 2000.

43. Sanders KM, Koh SD and Ward SM: Interstitial cells of Cajal as pacemakers in the gastrointestinal tract. Annu Rev Physiol 68: 307-343, 2006
44. Zhu MH, Kim TW, Ro S, Yan W, Ward SM, Koh SD and Sanders KM: A Ca ${ }^{2+}$-activated $\mathrm{Cl}^{-}$conductance in interstitial cells of Cajal linked to slow wave currents and pacemaker activity. J Physiol 587: 4905-4918, 2009.

(c) (i) (-) This work is licensed under a Creative Commons Attribution-NonCommercial-NoDerivatives 4.0 International (CC BY-NC-ND 4.0) License. 